\title{
Two different mutations in the envelope protein of feline immunodeficiency virus allow the virus to escape from neutralization by feline serum antibodies
}

\author{
Kees H.J. Siebelink ${ }^{\mathrm{a}}$, Marnix L. Bosch ${ }^{\mathrm{a}}$, Guus F. Rimmelzwaan ${ }^{\mathrm{b}}$, \\ Rob H. Meloen ${ }^{\mathrm{c}}$, Albert D.M.E. Osterhaus ${ }^{\mathrm{a}, \mathrm{b}, *}$ \\ ${ }^{2}$ Laboratory of Immunobiology, National Institute of Public Health and Environmental Protection, \\ Antonie van Leeuwenhoeklaan 9, PO Box 1,3720 BA Bilthoven, The Netherlands \\ 'Institute of Virology, Erasmus University Rotterdam, Dr. Molewaterplein 50, 3015 GE Rotterdam, \\ The Netherlands \\ 'Central Veterinary Institute, Edelherrweg 15, PO Box 65, 8200 AB Lelystad, The Netherlands
}

\begin{abstract}
Viral progeny of two molecular clones of feline immunodeficiency virus (FIV), $19 \mathrm{kl}$ and $19 \mathrm{k} 32$, were tested in a virus neutralization assay. In this assay the infection of thymocytes with FIV19k1 was neutralized by serum S1422, derived from an SPF cat 22 weeks after infection with FIV19k1. We previously reported that a point mutation at position 560 in hypervariable region-5 (HV-5) of $19 \mathrm{k} 1$ confers resistance to virus neutralization (Siebelink et al., 1993, J. Virol. 67: 2202-2208). Viral progeny of the other molecular clone, FIV19k32, which differs in the envelope protein in only six amino acids from $19 \mathrm{kl}$, was not neutralized. In order to map sites involved in virus neutralization we constructed chimeric clones by reciprocal exchange of $19 \mathrm{k} 1$ and $19 \mathrm{k} 32$ envelope gene fragments. Reciprocal exchange of a $1662 \mathrm{bp}$ fragment, encoding almost the whole surface protein, which differs in five amino acids between these two clones, resulted in exchange of the phenotype. Amino acids of the envelope protein of $19 \mathrm{k} 1$ and $19 \mathrm{k} 32$, in which these clones differ, were substituted by point mutation. We demonstrated that one of these mutations, a substitution of leucine to serine at position 483 in $\mathrm{HV}-4$, also conferred resistance of $19 \mathrm{k} 1$ to neutralization by serum S1422.
\end{abstract}

Abbreviations

FIV, feline immunodeficiency virus; HIV, human immunodeficiency virus; PBMC, peripheral blood mononuclear cells; SIV, simian immunodeficiency virus; TM, transmembrane; VN, virus neutralizing antibodies.

\footnotetext{
* Corresponding author at: Institute of Virology, Erasmus University Rotterdam, Dr. Molewaterplein 50, 3015 GE Rotterdam, The Netherlands Tel: (31) 10-4088066; Fax: (31) 10-4365145.
} 


\section{Introduction}

Virus neutralizing (VN) antibodies are generally accepted to play an important role in the protective immune response against infection with lentiviruses including human immunodeficiency virus (HIV) (Berman et al., 1990; Prince et al., 1991; Emini et al., 1992), simian immunodeficiency virus (SIV) (Lewis et al., 1993) and feline immunodeficiency virus (FIV) (Hohdatsu et al., 1993). However these VN antibodies are incapable of eliminating the virus from humans or animals. Infection thus results in persistence of the virus in the host leading to a continuous interaction between the virus and the immune system (Fauci, 1988). Genetic variation allows the virus to generate mutants which are no longer recognized by VN antibodies: so-called escape mutants. Such antigenic variation has been described in several lentiviral systems including Equine infectious anaemia virus (EIAV), Maedi-Visna virus, HIV and SIV (Narayan et al., 1977; Montelaro et al., 1984; Albert et al., 1990; Franchini et al., 1990; Burns et al., 1993; Joag et al., 1993). For HIV as well as for FIV it has been shown that one single point mutation in the envelope gene can confer resistance to virus neutralizing antibodies in vitro (Reitz et al., 1988, Siebelink et al., 1993). In the present study, it is shown that two independently arisen single point mutations in two different regions of the surface glycoprotein of FIV can each confer resistance to virus neutralizing antibodies present in a polyclonal serum. One of thesc mutations was generated in vitro whereas the other is observed in vivo.

\section{Materials and methods}

\subsection{Molecular clones and virus}

Replication competent molecular clones $19 \mathrm{k} 1$ and $19 \mathrm{k} 32$ were obtained directly from bone marrow cells of a naturally FIV infected cat as described previously (Siebelink et al., 1992). Viral progeny of $19 \mathrm{k} 1$ (FIV19k1) and 19k32 (FIV19k32) were obtained by transfection of Crandell feline kidney ( $\mathrm{CrFK}$ ) cells followed by cocultivation with feline peripheral blood mononuclear cells (PBMCs) as described previously (Siebelink et al., 1992). PBMCs infected with FIV19k1 were cultured in continuous presence of serum from Cat No. 14 (see below) to obtain the VN escape mutant as described previously (Siebelink et al., 1993).

\subsection{Sera}

A 10-week-old SPF cat (Cat No. 14) was infected with FIV19k1 by intraperitoneal inoculation of $10^{2} \mathrm{TCID}_{50}$. Serum of Cat No. 14 was taken prior to infection (preserum) and at 22 weeks post infection (designated serum S1422). Serum from a naturally FIV infected cat, A'dam 19 was designated serum AM19 (Siebelink et al., 1992). Serum of a non-infected SPF cat was used as a control serum (SPF serum). All sera were heat inactivated for $1 \mathrm{~h}$ at $56^{\circ} \mathrm{C}$ prior to use. 


\subsection{Reverse transcriptase assay}

Reverse transcriptase (RT) activity was determined as previously described (Siebelink et al., 1992).

\subsection{Virus neutralization assay}

VN antibodies were determined as previously described (Siebelink et al., 1993). In brief, $450 \mu \mathrm{l}$ of diluted virus stocks containing a RT activity of $5 \times 10^{4}$ counts per min per ml were incubated with $50 \mu \mathrm{l}$ of a serum sample. After $60 \mathrm{~min}$ at $37^{\circ} \mathrm{C} 500 \mu \mathrm{l}$ Con A and IL2 stimulated PBMC $\left(10^{6}\right)$ were added. After 12 days the culture supernatant was tested for presence of virus by RT activity. The virus neutralization index (VN index) was calculated by:

RT activity of the culture with SPF serum

RT activity of the culture with serum S1422

Virus neutralization was considered positive when the VN index was more then 3 , based on a survey of sera of non-infected cats (not shown).

\subsection{Sequence analysis}

The sequences of the envelope genes of the molecular clones $19 \mathrm{k} 1$ and $19 \mathrm{k} 32$ and of the VN escape mutant 19klesc have previously been described (Siebelink et al., 1992, 1993).

\subsection{Generation of chimeric clones}

Chimeric clones of $19 \mathrm{k} 1$ and $19 \mathrm{k} 32$ were constructed using the 5 '- and 3 '- subclone of $19 \mathrm{k} 1$ and $19 \mathrm{k} 32$ as described previously (Siebelink et al., 1993). Briefly, molecular clones of $19 \mathrm{kl}$ and $19 \mathrm{k} 32$ were digested with $S a I I$ and $S p h I$ and the fragments were subcloned. The 5' subclone contains gag and 5' part of pol and the $3^{\prime}$ subclone contain the $3^{\prime}$ part of $\mathrm{pol}$ and env. The chimeric clones are schematically represented in Fig. 1. Chimere 19k1560 is obtained by site directed mutagenesis as described previously for $19 \mathrm{k} 1$ mut 2 (Siebelink et al., 1993). The $3^{\prime}$ subclones of $19 \mathrm{k} 1$ and $19 \mathrm{k} 32$ were used to exchange internal 1662 bp $K p n I$ fragments between $19 \mathrm{k} 1$ and $19 \mathrm{k} 32$. These chimeras are designated $19 k 1 K K 32$ (19k1 containing the KpnI fragment of $19 \mathrm{k} 32$ ) and $19 \mathrm{k} 32 \mathrm{KK} 1$ (19k32 containing the $K p n I$ fragment of $19 \mathrm{k} 1$ ).

The 1662 bp KpnI fragment of 19k1 and 19k32 was subcloned into pUC19 (designated $3^{\prime} \mathrm{KK}$-subclone of $19 \mathrm{k} 1$ and $19 \mathrm{k} 32$, respectively) and used to exchange the $144 \mathrm{bp} \mathrm{Nsi}-$ Mst 2 fragment. These subclones were designated 19k1NM32 (19k1 containing the NsiI$M s t 2$ fragment of $19 \mathrm{k} 32$ ) and $19 \mathrm{k} 32 \mathrm{NM} 1$ ( $19 \mathrm{k} 32$ containing the NsiI-Mst 2 fragment of $19 \mathrm{k} 1$ ).

The $3^{\prime} \mathrm{KK}$-subclones of $19 \mathrm{k} 1$ and $19 \mathrm{k} 32$ were used for the substitution of single nucleotides by site directed mutagenesis using the transformer site directed mutagenesis kit (Clontech laboratories, USA). For the mutagenesis six oligonucleotides were designed. 
These oligonucleotides are listed in Table 1 . The mutagenesis was confirmed by sequence analysis.

The 1662 bp KpnI fragments from the $3^{\prime} \mathrm{KK}$ subclones of $19 \mathrm{k} 1$ and $19 \mathrm{k} 32$ in which 144 bp $N$ siI and $M s t 2$ fragments were exchanged or single nucleotides were mutated by site directed point mutation were cloned into the corresponding $3^{\prime}$ subclone of $19 \mathrm{k} 1$ and $19 \mathrm{k} 32$, respectively; $2.5 \mu \mathrm{g}$ of each of the $3^{\prime}$ subclones and the $5^{\prime}$ subclones were mixed, digested with $S p h I$ and ligated. After digestion with Sall the constructs were transfected into Crandell feline kidney cells (CrFK) as previously described (Siebelink et al., 1992). Virus production was rescued by cocultivation of transfected cells with Con A and IL-2 stimulated PBMC for 3 days. Cultures were monitored for syncytium formation of PBMC and RT activity in the culture supernatant. When RT activity could be measured culture supernatant was filtered through an $220 \mathrm{~nm}$ filter and stored in aliquots at $-135^{\circ} \mathrm{C}$.

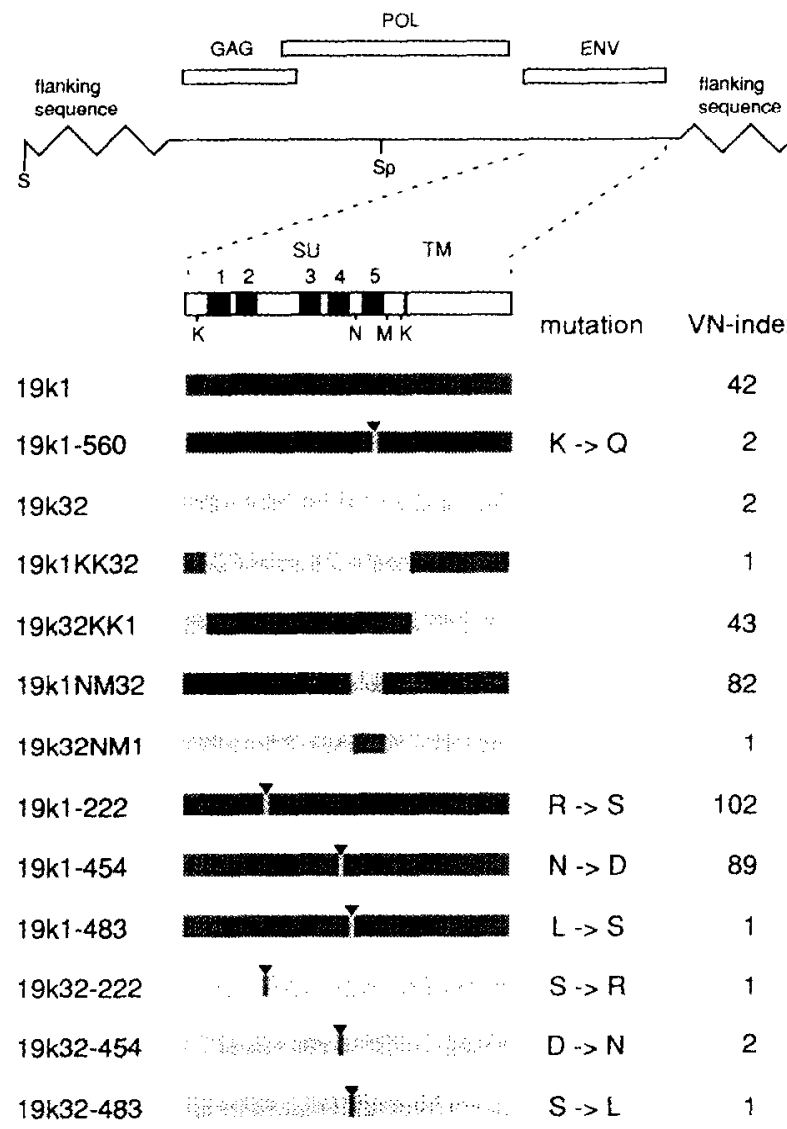

Fig. 1. Schematic representation of the chimeric FIV clones with the VN index (see text for details). The uppermost bars represent the major open reading frames gag, pol and env. The black boxes represent the hypervariable regions in the envelope protein. The patterns are corresponding to the parental clones. Individual point mutations are indicated with ' $\nabla$ '. Restriction enzyme cleaving sites are indicated as: K, KpnI; M, Mst $;$; N, Nsil; S, Sall; Sp, SphI. 
Table 1

Oligonucleotide sequences used for site directed mutagenesis

\begin{tabular}{|c|c|c|}
\hline Oligo & Sequence & Chimere name \\
\hline $1-666$ & $5^{\prime}$-CAAATATAAGTATACAAGAGG-3' & $19 \mathrm{kl}-222$ \\
\hline $1-1360$ & $5^{\prime}$-CAAAACATCATGATGAAGCAAGG-3' & $19 \mathrm{kl}-454$ \\
\hline $1-1448$ & 5'-CACAAAATGTTTCAGGTGC-3' & $19 \mathrm{k} 1-483$ \\
\hline $32-666$ & 5'-CAAATATAAGAATACAAGAGGG-3' & $19 \mathrm{k} 32-222$ \\
\hline $32-1360$ & 5'-CAAAACATCATAATGAAGCAAGG-3' & $19 \mathrm{k} 32-454$ \\
\hline $32-1448$ & 5'-CACAAAATGTTTTAGGTGC-3' & $19 \mathrm{k} 32-483$ \\
\hline
\end{tabular}

The mutations are indicated in bold.

\subsection{Synthesis and reaction of peptides with sera}

Twelve-mer peptides with an overlapping sequence of 11 amino acids were synthesized and analyzed for their reactivity with sera as previously described (Geijsen et al., 1984). The amino acid sequences of the peptides were based on the amino acid sequences of the envelope proteins of clones $19 \mathrm{kl}, 19 \mathrm{k} 32$ and $19 \mathrm{k}$ lesc between amino acid position 549 and 583 (HV-5) and between the amino acid positions 472 and 494 (HV-4). Three 12-mer control peptides with an overlapping sequence of 8 amino acids were synthesized according to the epitope between position 596 and 616 as previously described (Avrameas et al., 1992).

\section{Results}

\subsection{Neutralization of FIV19k1, FIVI9k32 and FIVI9klesc by serum $S 1422$}

Viral progeny of $19 \mathrm{k} 1$ and $19 \mathrm{k} 32$ and the escape mutant FIV19k1esc were tested in a VN assay with serum S1422. An SPF serum was used as a control. Virus replication as determined by RT activity was demonstrated in all cultures in the presence of SPF serum. In the presence of serum S1422 only in the cultures of FIV19k32 and FIV19k1esc and not in the culture of FIV19k1 virus replication could be demonstrated (Table 2).

\subsection{Sequence analysis}

Virus neutralizing antibodies which interfere with viral entry, are generally directed to the envelope proteins. Therefore the envelope protein sequences of FIV19k1, FIV19k32 and FIV19k1esc were compared. Amino acid sequence differences are listed in Table 3. Comparison of the envelope protein sequences of $19 \mathrm{k} 1$ and $19 \mathrm{k} 32$ showed the presence of six substitution mutations of which one is located in HV-4 and two are located in HV-5. The other three are found in the more conserved regions: one between HV-2 and HV-3, one between HV-4 and HV-5 and one in the transmembrane (TM) part of env. 


\subsection{Virus neutralization of chimeric clones}

Viral progeny of the parental clone $19 \mathrm{k} 1,19 \mathrm{k} 32$ and the chimeric clones were incubated with serum S1422 or SPF serum prior to infection of thymocytes. The results of this experiment are similar to a number of previous experiments in which each of the chimeric clones were tested separately for neutralization by serum S1422. RT activity was measured 12 days post infection. RT activity could be measured in all the control cultures. As shown previously (Siebelink et al., 1993) FIV19k1-560 was not neutralized by serum S1422 (Fig. 1). This virus has a lysine to glutamine substitution mutation at position 560 in HV-5.

Reciprocal exchange of a 1662 bp KpnI fragment between $19 \mathrm{k} 1$ and $19 \mathrm{k} 32$ resulted in the chimeric clones $19 \mathrm{k} 1 \mathrm{KK} 32$ and $19 \mathrm{k} 32 \mathrm{KK} 1$. Chimeric clone $19 \mathrm{k} 1 \mathrm{KK} 32$ was not neutralized by serum $\mathrm{S} 1422$ whereas chimeric clone $19 \mathrm{k} 32 \mathrm{KK} 1$ was neutralized by serum S1422 (Fig. 1 ) indicating that an epitope of the surface protein is involved in neutralization.

Reciprocal exchange of HV-5 by the exchange of the 144 bp NsiI-Mst 2 fragment resulted in the chimeric clones $19 \mathrm{k} 1 \mathrm{NM} 32$ (19k1 containing the $N s i I-M s t 2$ fragment of $19 \mathrm{k} 32$ ) and $19 \mathrm{k} 32 \mathrm{NM} 1$ ( $19 \mathrm{k} 32$ containing the $N s i \mathrm{I}-M s t 2$ fragment of $19 \mathrm{kl}$ ). Chimera $19 \mathrm{k} 1 \mathrm{NM} 32$ was neutralized with serum $S 1422$ whereas $19 \mathrm{k} 32 \mathrm{NM} 1$ was not neutralized with serum S1422 (Fig. 1) indicating that a site outside HV-5 is also involved in neutralization.

Therefore, the other three amino acids which are different between $19 \mathrm{kl}$ and $19 \mathrm{k} 32$ were substituted separately by site directed mutagenesis. The mutated clones of $19 \mathrm{k} 1$ were designated 19k1-222 (position 222, Arg to Ser), 19k1-454 (position 454, Asn to Asp)

Table 2

Virus neutralization of molecular clones $19 \mathrm{kl}$ and $19 \mathrm{k} 32$ and the VN escape mutant $19 \mathrm{klesc}$ with serum S1422 and SPF serum

\begin{tabular}{lrrr}
\hline Virus & \multicolumn{2}{c}{ RT activity after neutralization with } & VN index \\
\cline { 2 - 3 } & Serum S1422 & SPF serum & 48 \\
\hline FIV19k1 & 3636 & 174990 & 1 \\
FIV19k1esc & 65925 & 52845 & 1 \\
FIV19k32 & 104658 & 58782 & 1 \\
\hline
\end{tabular}

Table 3

Differences between $19 \mathrm{k} 1,19 \mathrm{k} 32$ and $19 \mathrm{k}$ lesc in the envelope protein

\begin{tabular}{lllll}
\hline HV-region & Position & $19 \mathrm{k} 1$ & $19 \mathrm{k} 32$ & $19 \mathrm{klesc}$ \\
\hline $2-3$ & 222 & Arg & Ser & Arg \\
4 & 454 & Asn & Asp & Asn \\
$4-5$ & 483 & Leu & Ser & Leu \\
5 & 555 & Asn & Lys & Asn \\
5 & 556 & Asn & Asn & Thr \\
5 & 557 & His & Tyr & His \\
5 & 560 & Lys & Lys & Gln \\
TM & 741 & Ala & Asp & Ala \\
\hline
\end{tabular}


and $19 \mathrm{k} 1-483$ (position 483, Leu to Ser). The corresponding clones of $19 \mathrm{k} 32$ were reciprocally substituted and designated accordingly. FIV 19k1-222 and FIV 19k1-454 were neutralized by serum $S 1422$ whereas FIV $19 \mathrm{k} 1-483$ could not be neutralized indicating that the leucine to serine substitution at position 483 can also confer resistance to virus neutralization by serum S1422. FIV 19k32-222, FIV 19k32-454 and FIV19k32-483 could not be neutralized by serum $S 1422$.

\subsection{Pepscan analysis}

Twelve-mer peptides representing the amino acid sequence of the clones $19 \mathrm{kl}$ and the escape mutant $19 \mathrm{klesc}$ between amino acid positions 549 and 583 and between the amino acid positions 472 and 494 were analyzed for reactivity with serum S1422, serum from a naturally FIV infected cat Adam19 (serum AM19) and SPF serum. As a control for the pepscan procedure three 12-mer peptides spanning, with an overlap of eight amino acids, a previously described linear epitope on the envelope protein of FIV (from amino acid position 596 to 616) were scanned with serum S1422, serum AM19 and SPF serum. No reactivity in these sera was detected with the HV-5 peptides and HV-4 peptides whereas serum S1422 and serum AM19 did react in the control pepscan (not shown) suggesting that no linear B-cell epitope in the C-terminal half of HV-5 is recognized by serum S1422 and serum AM19.

\section{Discussion}

In the present report we have demonstrated that two independent single amino acid substitutions can confer resistance to virus neutralizing antibodies present in a polyclonal cat serum. Neither of the two regions in which these mutations occurred demonstrated reactivity with this serum in a pepscan analysis. This indicates that the recognized epitope( $\mathrm{s}$ ) cannot be mimicked with linear synthetic peptides and consequently that the recognized epitopes are by themselves not linear. The finding that both mutations, occurring at two regions in the surface glycoprotein that are approximately 80 amino acids apart, lead to escape from neutralization would then indicate that these regions may well be involved in the formation of a single conformational epitope, in which they would be in close proximity to each other spatially. Point mutations at each of these regions would disturb the conformation, resulting in loss of binding by the antibodies present in the serum that recognize the aforementioned conformational epitope. An alternative explanation would be, that both the mutations caused a major change in the overall conformation of the surface glycoprotein thus allowing a number of different VN epitopes to change their conformation or to hide from recognition by VN antibodies. Serum S1422 was collected from Cat No, 14 at 5 months post infection with FIV19k1. Using our system we can only find evidence for the existence of one major neutralization epitope. However, this finding does not preclude the presence of VN antibodies reactive to other envelope regions which may be detected in other or modified culture systems. It is interesting to note that the two regions in which the mutations have been observed are both located in the $\mathrm{C}$-terminal half of the surface glycoprotein. For HIV-1 it has been demonstrated that it binds its cellular receptor CD4 with a 
conformationally formed hinding site, to which especially regions at the C-terminal half of gp120 contribute. Antibodies directed at this conformational CD4-binding site neutralize HIV-1. It is conceivable that our data, indicating the presence of a conformational virus neutralizing epitope in the C-terminal half of the surface glycoprotein, likewise point to the presence of a similar receptor binding site formed in this region of the FIV envelope.

The change in VN phenotype between the progenies of clones $19 \mathrm{k} 1$ and $19 \mathrm{k} 32$, which could be mimicked by introducing only one of the naturally occurring genetic differences between these clones namely a leucine to serine substitution at position 483, suggests that $19 \mathrm{k} 32$ has resulted from a mutant of FIV that had escaped from antibodies that neutralized FIV19k1. Clones 19k1 and 19k32 coexisted in the bone marrow cells of cat AM19 (Siebelink et al., 1992). The viral progeny of these clones exhibit differential susceptibilities to $\mathrm{VN}$ antibodies which indicates that the generation of VN escape mutants is an ongoing process in vivo in FIV infected cats. The fact that we could not make FIV 19k32 susceptible to $\mathrm{VN}$ by serum $S 1422$ through the serine to leucine substitution at position 483 indicates that these two viruses differ from each other at more than one position contributing to the pertinent conformational neutralizing epitope. A combination of two or more mutations may induce VN susceptibility in FIV $19 \mathrm{k} 32$. This work is presently in progress.

The leucine to serine substitution mutation at position 483 introduces a potential $\mathrm{N}$-linked glycosylation site at position 481 . N-linked glycosylation has been postulated to mask viral neutralizing epitopes (Alexander and Elder, 1984) or hinder binding of VN antibodies (Davis et al., 1990). The above postulated conformation of the VN epitope could be masked by the introduced carbohydrate group or the carbohydrate group can actually by itself change the conformation of the VN epitope. Genetic exchange experiments and point mutations will reveal whether the $\mathrm{N}$-linked glycan plays indeed a role in the escape mechanism.

\section{Acknowledgements}

We thank C. Kruyssen for preparing the manuscript and W. Puyk for performing the pepscan analysis. This work was supported by the Advisory Council on Health Research (RGO No. 88-90/89028) and the concerted action on feline AIDS of the EC.

\section{References}

Albert, J., Abrahamsson, B., Nagy, K., Aurelius, E., Gaines, H., Nyström, G. and Fenyö, M., 1990. Rapid development of isolate-specific neutralizing antibodies after primary HIV-1 infection and consequent emergence of virus variants which resist neutralization by autologous sera. AIDS, 4: 106-112.

Alexander, S. and Elder, J.H., 1984. Carbohydrate dramatically influences immune reactivity of antisera to viral glycoprotein antigens. Science, 226: 1328-1330.

Avrameas, A., Guillet, I., Chouchane, L., Moraillon, A., Sonigo, P. and Strosberg, A.D., 1992, Localization of three epitopes of the env protein of feline immunodeficiency virus. Mol. Immunol., 29: 565-572.

Berman, P., Gregory, T., Ridale, L., Nakamura, G., Champe, M., Porter, J., Wurm, F., Herscberg, R., Cobb, E.K. and Eicbberg, J., 1990. Protection of chimpanzees from infection by HIV-1 after vaccination with recombinant glycoprotein gp 120 but not gp 160. Nature, 345: 622-625.

Burns, D.P.W., Collignon, C. and Desrosicrs, R.C., 1993. Simian immunodeficiency virus mutants resistant to serum neutralization arise during persistent infection of rhesus monkeys. J. Virol., 67: 4104-4113. 
Davis, D., Stephens, D.M., Willers, C. and Lachmann, P.J., 1990. Glycosylation governs the binding of antipeptide antibodies to regions of hypervariable amino acid sequence within recombinant gp 120 of human immunodeficiency virus type 1. J. Gen. Virol., 71: 2889-2898.

Emini, E.A., Schleif, W.A., Nunberg, J.H., Conley, A.J., Eda, Y., Tokiyoshi, S., Putney, S.D., Matsushita, S., Cobb, K.E., Jett, C.M., Eichberg, J.W. and Murthy K.K., 1992. Prevention of HIV-l infection in chimpanzees by gp $120 \mathrm{~V} 3$ domain-specific monoclonal antibody. Nature, 355: 728-730.

Fauci, S.A., 1988. The human immunodeficiency virus: infectivity and mechanism of pathogenesis. Science, 239: $617-622$.

Franchini, G., Markham, P., Gard, E., Fargnoli, K., Keubaruwa, S.. Jagodzini. L., Robert-Guroff, M.. Lusso, P., Ford, G.. Wong-Staal, F. and Gallo, R.C., 1990. Persistent infection of thesus macaques with a molecular clone of human immunodeficiency virus type 2: evidence of minimal genetic drift and low pathogenic effects. J. Virol., 64: 4462-4467.

Geijsen, H.M., Meloen, R.H. and Barteling, S.J., 1984. Use of peptide synthesis to probe viral antigens for epitopes to a resolution of a single amino acid. Proc. Natl. Acad. Sci. USA, 81: 3998-4002.

Hohdatsu, T., Pu, R., Torres, B.A., Trujillo, S., Gardner, M.B. and Yamamoto, J.K., 1993. Passive antibody protection of cats against feline immunodeficiency vinus infection. J. Virol., 67: 2344-2348.

Joag, S.V., Anderson, M.G., Clements, J.E., McEntee, M.F., Sharma, D.P., Adams, R.J. and Narayan, O.. 1993. Antigenic variation of molecularly cloned SIV $_{\text {mac }} 239$ during persistent infection in a rhesus macaque. Virology, 195: 406-412.

Lewis, M.G., Elkins, W.R., McCutchan, F.E., Benveniste, R.E., Lai, C.Y., Montefori, D.C., Burke, D.S., Eddy, G.A. and Shafferman, A., 1993. Passively transferred antibodies directed against conserved regions of SIV envelope protect macaques from SIV infection. Vaccine, 11: 1347-1355.

Montelaro, R.C., Parekh, B., Orrego, A. and Issel, J., 1984. Antigenic variation during persistent infection by equine infectious anaemia virus, a retrovirus. J. Biol. Chem., 256: 10539-10544.

Narayan, O., Griffin, D.E. and Chase, J., 1977. Antigenic shift of visna virus in persistently infected sheep. Science, 197: 376-378.

Prince, A.M., Reesink, H., Pascual, D., Horowitz, B., Hewlett, 1., Murthy, K.K., 1991. Prevention of HIV infection by passive immunization with HIV immunoglobulin. Aids Res. Hum. Retroviruses, 7: 971-973.

Reitz, M.S., Wilson, C., Naugle, C., Gallo, R.C. and Robert-Guroff, M., 1988. Generation of a neutralizationresistant variant of HIV-1 is due to selection for a point mutation in the envelope gene. Cell, 54: 57-63.

Siebelink, K.H.J., Chu, I., Rimmelzwaan, G.F., Weijer, K., Osterhaus, A.D.M.E. and Bosch., M.L., 1992. Isolation and partial characterization of infectious molecular clones of feline immunodeficiency virus obtained directly from bone marrow DNA of a naturally infected cat. J. Virol., 66: 1091-1097.

Siebelink, K.H.J., Rimmelzwaan, G.F., Bosch, M.L., Meloen, R.H. and Osterhaus, A.D.M.E., 1993, A single amino acid substitution in hypervariable region 5 of the envelope protein of feline immunodeficiency virus allows escape from virus neutralization. J. Virol., 67: 2202-2208. 